University of Nebraska - Lincoln

DigitalCommons@University of Nebraska - Lincoln

March 2008

\title{
Structures and relative stability of medium- and large-sized silicon clusters. VI. Fullerene cage motifs for low-lying clusters Si39, Si40, Si50, Si $60, \mathrm{Si}_{70}$, and $\mathrm{Si}_{80}$
}

Soohaeng Yoo

University of Nebraska-Lincoln

N. Shao

University of Nebraska-Lincoln

Xiao Cheng Zeng

University of Nebraska-Lincoln, xzeng1@unl.edu

Follow this and additional works at: https://digitalcommons.unl.edu/chemzeng

Part of the Chemistry Commons

Yoo, Soohaeng; Shao, N.; and Zeng, Xiao Cheng, "Structures and relative stability of medium- and largesized silicon clusters. VI. Fullerene cage motifs for low-lying clusters Si39, Si40, Si50, Si60, Si70, and Si80" (2008). Xiao Cheng Zeng Publications. 86.

https://digitalcommons.unl.edu/chemzeng/86

This Article is brought to you for free and open access by the Published Research - Department of Chemistry at DigitalCommons@University of Nebraska - Lincoln. It has been accepted for inclusion in Xiao Cheng Zeng Publications by an authorized administrator of DigitalCommons@University of Nebraska - Lincoln. 


\title{
Structures and relative stability of medium- and large-sized silicon clusters. VI. Fullerene cage motifs for low-lying clusters $\mathrm{Si}_{39}, \mathrm{Si}_{40}, \mathrm{Si}_{50}, \mathrm{Si}_{60}, \mathrm{Si}_{70}$, and $\mathrm{Si}_{80}$
}

\author{
Soohaeng Yoo, N. Shao, and X. C. Zeng ${ }^{\text {a) }}$ \\ Department of Chemistry and Center for Materials and Nanoscience, University of Nebraska-Lincoln, \\ Lincoln, Nebraska 68588, USA
}

(Received 5 November 2007; accepted 16 January 2008; published online 13 March 2008)

\begin{abstract}
We performed a constrained search, combined with density-functional theory optimization, of low-energy geometric structures of silicon clusters $\mathrm{Si}_{39}, \mathrm{Si}_{40}, \mathrm{Si}_{50}, \mathrm{Si}_{60}, \mathrm{Si}_{70}$, and $\mathrm{Si}_{80}$. We used fullerene cages as structural motifs to construct initial configurations of endohedral fullerene structures. For $\mathrm{Si}_{39}$, we examined six endohedral fullerene structures using all six homolog $\mathrm{C}_{34}$ fullerene isomers as cage motifs. We found that the $\operatorname{Si}_{39}$ constructed based on the $\mathrm{C}_{34}\left(C_{s}: 2\right)$ cage motif results in a new leading candidate for the lowest-energy structure whose energy is appreciably lower than that of the previously reported leading candidate obtained based on unbiased searches (combined with tight-binding optimization). The $\mathrm{C}_{34}\left(C_{S}: 2\right)$ cage motif also leads to a new candidate for the lowest-energy structure of $\mathrm{Si}_{40}$ whose energy is notably lower than that of the previously reported leading candidate with outer cage homolog to the $\mathrm{C}_{34}\left(C_{1}: 1\right)$. Low-lying structures of larger silicon clusters $\mathrm{Si}_{50}$ and $\mathrm{Si}_{60}$ are also obtained on the basis of preconstructed endohedral fullerene structures. For $\mathrm{Si}_{50}, \mathrm{Si}_{60}$, and $\mathrm{Si}_{80}$, the obtained low-energy structures are all notably lower in energy than the lowest-energy silicon structures obtained based on an unbiased search with the empirical Stillinger-Weber potential of silicon. Additionally, we found that the binding energy per atom (or cohesive energy) increases typically $>10 \mathrm{meV}$ with addition of every ten $\mathrm{Si}$ atoms. This result may be used as an empirical criterion (or the minimal requirement) to identify low-lying silicon clusters with size larger than $\mathrm{Si}_{50}$. (C) 2008 American Institute of Physics. [DOI: 10.1063/1.2841080]
\end{abstract}

\section{INTRODUCTION}

The notion of endohedral silicon fullerenes was first put forward in the early 1990s. ${ }^{1-7}$ Over the past few years, medium-sized endohedral silicon fullerenes have received increasing attention ${ }^{8-22}$ largely because of the advancement of global optimization methods and their application to medium-sized silicon clusters. ${ }^{23-27}$ Numerous theoretical studies of lowest-energy structures of medium-sized clusters $\mathrm{Si}_{N}(N=25-40$ and 45$)$ have shown that beyond the size $N$ $=29$, spherical-like compact structures are more stable than elongated structures $9,13,15,16,20$ and that the carbon fullerene cages are likely to be generic cage motifs for a class of lowlying spherical-like clusters. ${ }^{9,14,16-18,20,21}$ An empirical rule for determining optimal "stuffing@cage" combination for endohedral silicon fullerenes has been proposed.", $\mathrm{Re}$ cently, exploration of low-lying endohedral silicon fullerenes has been extended to silicon clusters beyond the size $N=45,{ }^{20,21}$ such as $\mathrm{Si}_{60},{ }^{10,20-22}$ for which a great deal of theoretical effort has been made due to its relevance to the "bucky silicon" $\mathrm{Si}_{60}{ }^{28-37}$

In our previous study (Paper $\mathrm{V}$ in this series ${ }^{17}$ ), we have shown that candidates for the lowest-energy structures of silicon clusters $\operatorname{Si}_{N}(N=31-40)$ are all in the form of endohedral fullerenes except $\mathrm{Si}_{39}$, whose outer cage contains a seven-member ring. In that study, we used an unbiased glo-

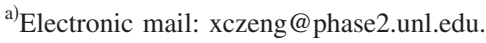

bal search method (the minima-hopping method ${ }^{38}$ ) directly combined with the semiempirical density-functional based tight-binding (DFTB) model of silicon. ${ }^{39}$ Since the number of possible low-lying isomers grows dramatically with increase of cluster size, it becomes impossible to locate the global minima on the potential energy surface (PES) for medium- to large-sized silicon clusters. The unconstrained (global) search combined with the DFTB model has also become increasingly computationally demanding. It will be desirable to obtain some candidates of low-lying isomers before launching such a computationally demanding unconstrained global search. The motif-based constrained search is an option to achieve representative low-lying clusters. On one hand, motif-based search is highly efficient since the selection of motif dramatically reduces the dimensions of the potential energy surface. On the other hand, this approach is capable of producing competitive candidates for the lowlying clusters, as has been demonstrated for smaller silicon clusters $\mathrm{Si}_{N}$ in the size range of $N=15-30 .{ }^{13,17}$

In view of several previous studies (using various unconstrained global search methods) confirming that fullerenes are viable structural motifs to build low-lying medium-sized silicon clusters, here, we further examine fullerene-based structural motifs for constructing medium- to large-sized low-lying silicon clusters. To this end, we have performed a series of constrained (biased) searches using carbon fullerenes as cage motifs to seek low-lying silicon clusters of $\mathrm{Si}_{50}, \mathrm{Si}_{60}, \mathrm{Si}_{70}$, and $\mathrm{Si}_{80}$. To validate this approach, we used 
$\mathrm{Si}_{39}$ and $\mathrm{Si}_{40}$ as testing bed. Interestingly, for $\mathrm{Si}_{39}$, we have found that a number of endohedral fullerene structures are appreciably lower in energy than the nonfullerene $\mathrm{Si}_{39}$ structure reported by us in Paper V. ${ }^{17}$ For $\mathrm{Si}_{50}$ and $\mathrm{Si}_{60}$, we have found new low-energy endohedral fullerene structures whose energies are notably lower than those previously reported in the literature. ${ }^{12,21,22}$ Finally, we obtain low-energy endohedral fullerene structures for $\mathrm{Si}_{70}$ and $\mathrm{Si}_{80}$. The structures of $\mathrm{Si}_{50}, \mathrm{Si}_{60}$, and $\mathrm{Si}_{80}$ may be considered as low-lying structures because their energies are appreciably lower than those of the corresponding low-lying structures obtained based on the unbiased global search with the Stillinger-Weber potential of silicon.

\section{COMPUTATIONAL METHOD}

Geometric optimization for all clusters was carried out using the plane-wave-pseudopotential density-functional theory (PWP-DFT) within generalized-gradient approximation (GGA). Two GGA functionals were employed (see reason below), the Becke exchange and Lee-Yang-Parr correlation $^{40}$ (BLYP) and the Perdew-Burke-Ernzerhof (PBE) functional, ${ }^{41}$ both implemented in the CPMD program. ${ }^{42}$ In the PWP-DFT calculations, we used an energy cutoff of $30 \mathrm{Ry}$ for plane-wave expansion and a supercell size of $25 \AA$ in dimension.

We employed the basin-hopping (BH) global optimization technique ${ }^{43}$ combined with the PWP-DFT optimization to search for low-energy clusters. ${ }^{27}$ The BH method essentially converts the potential energy surface $(\widetilde{E})$ of a cluster to a multidimensional "staircase" via the mapping $\widetilde{E}(\mathbf{X})$ $=\min \{E(\mathbf{X})\}$, where $\mathbf{X}$ denotes the nuclear coordinates of the cluster and "min" refers to the energy minimization performed starting from $\mathbf{X}{ }^{43}$ Specifically, the canonical Monte Carlo (MC) sampling method was used to sample the transformed potential energy surface $\widetilde{E}$ at a fixed temperature. With each MC move in the BH search, coordinates of all core atoms are randomly displaced while those of the outercage atoms are constrained, followed by a geometry optimization using the PWP-DFT methods. In the BH search, we used a smaller energy cutoff (12 Ry) for plane-wave expansion and a smaller supercell size (15 $\AA$ ). For the purpose of comparison, we also used the unbiased $\mathrm{BH}$ global optimization method to locate the lowest-energy clusters of $\mathrm{Si}_{39}, \mathrm{Si}_{40}$, and $\mathrm{Si}_{50}$ based on an empirical Stillinger-Weber (SW) potential of silicon. ${ }^{44,45}$

In previous studies of small-sized clusters $\mathrm{Si}_{N}(N$ =7-20) (Papers I-III, Refs. 13, 46, and 47), we used $a b$ initio molecular-orbital methods at the MP2/6-31G $(d)$ level of theory for geometry optimization and the coupled-cluster theory for total-energy calculation. However, for medium- to large-sized silicon clusters, geometry optimization at the MP2/6-31G $(d)$ level is computationally very demanding, and the total-energy calculation at the coupled-cluster level of theory is impractical. Therefore the calculation of binding energies per atom and determination of the energy ranking among isomers of silicon clusters are all based on densityfunctional level of theory. Since DFT entails a larger error bar in total-energy calculation compared to the MP2 or coupled-cluster methods, we thus view those isomers with binding energy differences less than $4 \mathrm{meV}$ as isoenergetic. It is known that the energy ranking among isomers with very close energy can be sensitive to the functional selected in the DFT calculation. Moreover, we have found that the PBE functional favors more compact silicon clusters (in terms of energy ranking) whereas the BLYP functional favors clusters having less core-filling atoms. ${ }^{13,17}$ We thus present results of binding energies based on the two functionals, namely, the PBE and BLYP.

\section{RESULTS AND DISCUSSIONS}

Initial configurations of endohedral fullerene clusters were constructed using carbon fullerenes ${ }^{48,49}$ as cage motifs, in which a certain number of core-filling silicon atoms were added randomly. The selection of the number of core-filling atoms and the associated fullerene cage motif was guided based on an empirical rule derived previously to achieve optimal core-filling@cage combination. ${ }^{9}$ Specifically, the $\mathrm{Si}_{m+3} / \mathrm{Si}_{m}(m=1,2, \ldots)$ is an upper/lower limit for the number of core-filling atoms to fit in a $\mathrm{Si}_{26+2 m}$ fullerene cage. For example, to construct an endohedral fullerene cluster $\mathrm{Si}_{40}$, an optimal core-filling@cage combination is $\mathrm{Si}_{6} @ \mathrm{Si}_{34}$ whereas $\mathrm{Si}_{8} @ \mathrm{Si}_{32}$ or $\mathrm{Si}_{4} @ \mathrm{Si}_{36}$ combination may be slightly "overstuffing" or "understuffing" according to the empirical rule.

\section{A. $\mathrm{Si}_{39}\left(\mathbf{S i}_{5} @ \mathrm{Si}_{34}\right)$}

In Paper $\mathrm{V},{ }^{17}$ we reported a low-lying $\mathrm{Si}_{39}$ cluster with an outer cage of $\mathrm{Si}_{34}$. However, the outer cage $\mathrm{Si}_{34}$ contains a seven-membered ring, and thus is not a classical fullerene. Moreover, the cluster has less binding energy per atom than its neighbor low-lying clusters $\mathrm{Si}_{38}$ and $\mathrm{Si}_{40}$, indicating that $\mathrm{Si}_{39}$ cluster is not the best candidate for the lowest-energy cluster. Later, Ma et al. ${ }^{18}$ reported an endohedral fullerene the $\mathrm{Si}_{39}$ structure with a slightly larger binding energy per atom in DFT-PBE calculation than that reported in Ref. 17. The homolog carbon cage of this endohedral fullerene $\mathrm{Si}_{39}$ structure is $\mathrm{C}_{34}\left(C_{s}: 2\right)$ in Fowler-Manolopoulos fullerene notation (where $C_{s}$ refers to the point-group symmetry and 2 refers to the Fowler-Manolopoulos label). ${ }^{48}$

To achieve an improved endohedral fullerene cluster of $\mathrm{Si}_{39}$, we examined all six isomers of fullerene $\mathrm{C}_{34}$ as cage motifs for initial configurations. We performed $\mathrm{BH}$ search based on the six initial endohedral fullerene structures. The obtained six low-energy structures are labeled as si39-1a to si39-1f (Fig. 1), in the rank of decreasing binding energy per atom (Table I). Among the six $\mathrm{Si}_{39}$ isomers, the homolog fullerene cage of si39-1a and si39-1b is $\mathrm{C}_{34}\left(C_{s}: 2\right)$ and $\mathrm{C}_{34}\left(C_{2}: 5\right)$, respectively. Both si39-1a and si39-1b are notably lower in energy than those reported previously. ${ }^{17,18}$ si39-1a has a binding energy per atom of $3.959 \mathrm{eV}$ (PBE calculation), about $10 \mathrm{meV}$ greater than that of si39-1b. More importantly, si39-1a has a greater binding energy per atom $(\mathrm{PBE})$ than that $(3.948 \mathrm{eV})$ of the leading low-lying structure of $\mathrm{Si}_{38}{ }^{17}$ si39-1a also has a binding energy per atom of $3.364 \mathrm{eV}$ (BLYP calculation), which is nearly the same as that reported in Ref. $17(3.365 \mathrm{eV})$. In summary, si39-1a is definitely qualified as a low-lying cluster and is 


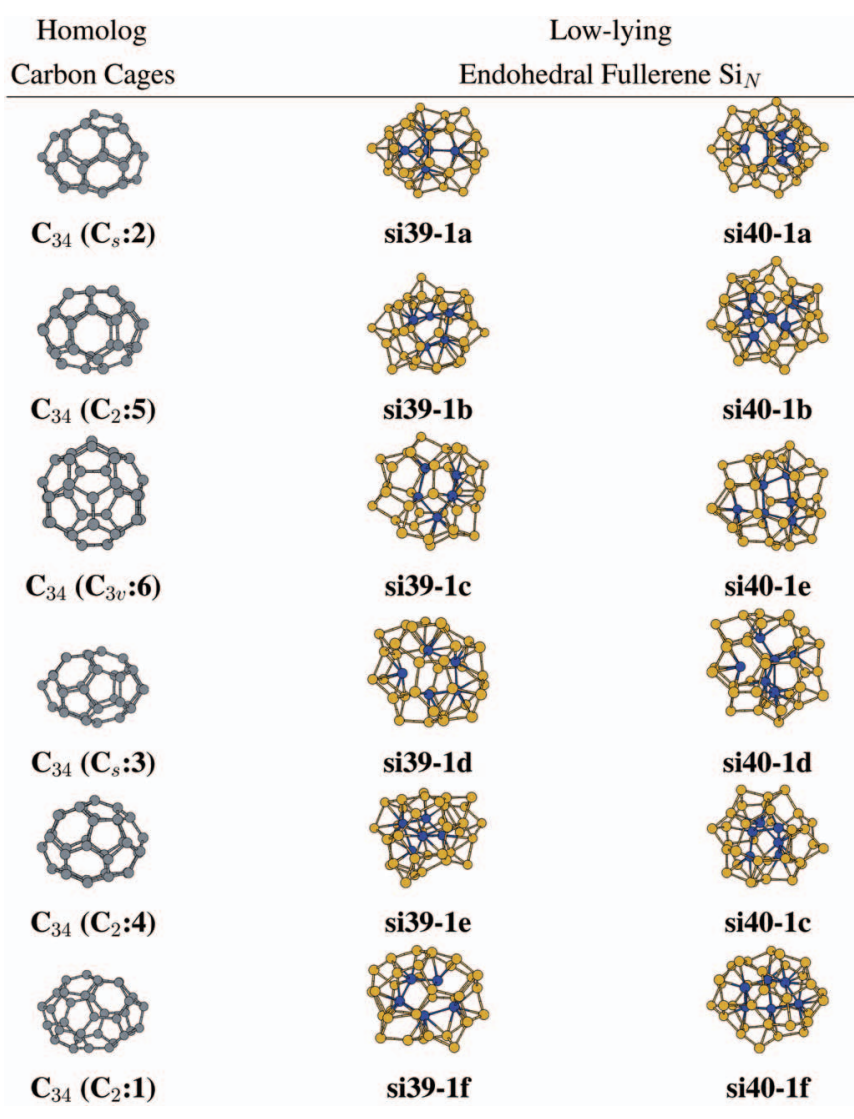

FIG. 1. (Color) Geometries of low-lying endohedral silicon fullerene clusters $\mathrm{Si}_{39}$ and $\mathrm{Si}_{40}$. The core-filling atoms are in blue and the outer cage atoms are in yellow. The corresponding homolog carbon fullerene cages (gray) are also displayed.

currently the new leading candidate for the lowest-energy structure of $\mathrm{Si}_{39}$. This structure can be used in the future study of the chemical reactivity of $\mathrm{Si}_{39}$ with small chemical molecules such as $\mathrm{C}_{2} \mathrm{H}_{2}$ and $\mathrm{NO}_{2}$. ${ }^{50}$

\section{B. $\mathrm{Si}_{40}\left(\mathrm{Si}_{6} @ \mathrm{Si}_{34}\right.$ or $\left.\mathrm{Si}_{4} @ \mathrm{Si}_{36}\right)$}

In Ref. 12, we reported a low-lying endohedral fullerene structure of $\mathrm{Si}_{40}$ which has a binding energy per atom of 3.943 eV (PBE calculation, Table I). In Ref. 17, we reported another low-lying endohedral fullerene structure of $\mathrm{Si}_{40}$ which has a binding energy per atom $(3.372 \mathrm{eV})$ in BLYP calculation. The homolog fullerene cage of the former $\mathrm{Si}_{40}$ structure is $\mathrm{C}_{34}\left(C_{3 v}: 6\right)$, whereas that of the latter is $\mathrm{C}_{36}\left(D_{3 h}: 13\right)$ (Table I). As shown in previous studies, ${ }^{13,17}$ the PBE functional favors more compact clusters, whereas the BLYP functional favors clusters having a larger fullerene cage and less core-filling atoms.

Zhou and Pan recently reported a new low-lying structure of $\mathrm{Si}_{40}$. They used the "compressing-liquid-silicon" strategy together with the stuffed-fullerene approach. ${ }^{21}$ The outer cage of the new $\mathrm{Si}_{40}$ structure is homolog to a $\mathrm{C}_{34}$ cage having a seven-member ring. However, the binding energy per atom (in PBE calculation) is $3.955 \mathrm{eV}$, appreciably larger than that reported in Ref. 12. We have tested whether the fullerene cage motifs can outperform the nonfullerene cage motifs to obtain new $\mathrm{Si}_{40}$ with lower energies. Again, we used all six isomers of fullerene $\mathrm{C}_{34}$ as cage motifs to con- struct initial endohedral fullerene configurations of $\mathrm{Si}_{40}$. Expectedly, two new endohedral fullerene structures of $\mathrm{Si}_{40}$ are obtained, namely, si40-1a and si40-1b (Fig. 1). Both si40-1a and si40-1b have binding energy per atom (3.966 and $3.962 \mathrm{eV}$ in PBE calculation or 3.364 and $3.365 \mathrm{eV}$ in BLYP calculation; see Table I) notably greater than the best candidate $(3.955 \mathrm{eV}$ in $\mathrm{PBE}$ binding energy) reported previously. $^{21}$ Interestingly, si40-1a possesses the same homolog fullerene cage $\mathrm{C}_{34}\left(C_{s}: 2\right)$ as si39-1a while si40-1b possesses the same homolog fullerene cage $\mathrm{C}_{34}\left(C_{2}: 5\right)$ as si39-1b, suggesting that the fullerene cages $\mathrm{C}_{34}\left(C_{s}: 2\right)$ and $\mathrm{C}_{34}\left(C_{2}: 5\right)$ are the two best cage motifs to generate lowerlying $\mathrm{Si}_{39}$ and $\mathrm{Si}_{40}$. Note also that the leading candidate for the lowest-energy structure of $\mathrm{Si}_{38}$ (Paper V, Ref. 17) also possesses the homolog $\mathrm{C}_{34}\left(C_{2}: 5\right)$ cage. In summary, si40-1a and si40-1b are new leading candidates for the lowest-energy structure of $\mathrm{Si}_{40}$ based on PBE calculation, whereas si40-1a' (Table I) ${ }^{17}$ remains to be the leading candidate based on BLYP calculation. si40-1 $\mathrm{a}^{\prime}$ possesses an outer cage homolog to $\mathrm{C}_{36}\left(D_{3 h}: 13\right)$.

\section{C. $\mathrm{Si}_{50}\left(\mathrm{Si}_{8} @ \mathrm{Si}_{42}\right.$ or $\left.\mathrm{Si}_{6} @ \mathrm{Si}_{44}\right)$}

According to the empirical rule illustrated previously, an optimal core-filling@cage combination for endohedral fullerene $\mathrm{Si}_{50}$ is $\mathrm{Si}_{8} @ \mathrm{Si}_{42}$ or $\mathrm{Si}_{6} @ \mathrm{Si}_{44}$. In the previous study, ${ }^{12}$ we performed an unbiased search for the global minimum of $\mathrm{Si}_{50}$ using the generic algorithm method combined with the nonorthogonal tight-binding model for silicon. ${ }^{51}$ Interestingly, the unbiased search showed that the lowest-energy structure of $\mathrm{Si}_{50}$ is an endohedral fullerene whose outer cage is homolog to $\mathrm{C}_{42}\left(C_{1}: 39\right)$ [Fig. 2(a)]. The binding energy per atom is $3.954 \mathrm{eV}$ (PBE calculation; Table II), less than that of the low-lying structures of $\mathrm{Si}_{39}$ and $\mathrm{Si}_{40}$. In this sense, this $\mathrm{Si}_{50}$ structure may no longer be considered as a low-lying structure. Zhou and Pan reported another lowenergy structure of $\mathrm{Si}_{50}$ using the compressing-liquid-silicon approach. ${ }^{21}$ The outer cage of that $\mathrm{Si}_{50}$ is homolog to a $\mathrm{C}_{40}$ cage containing a seven-member ring. Its binding energy per atom is $3.950 \mathrm{eV}$ (PBE calculation; Table II), less than that reported in Ref. 12. Thus, it seems that the $\mathrm{Si}_{10} @ \mathrm{Si}_{40}$ combination is slightly overstuffing for $\mathrm{Si}_{50}$.

Here, we constructed two initial configurations of endohedral fullerene $\mathrm{Si}_{50}$ using the lowest-energy carbon fullerene $\mathrm{C}_{42}\left(D_{3}: 45\right)$ and $\mathrm{C}_{44}\left(D_{2}: 75\right)$ as cage motifs. ${ }^{47} \mathrm{We}$ then performed a $\mathrm{BH}$ search combined with the DFT/PBE optimization for the former and with the DFT/BLYP optimization for the latter. Both $\mathrm{BH}$ searches give two markedly improved endohedral fullerene structures, namely, si50-1a and si50-1a' [Fig. 2(a)]. The si50-1a is particularly noteworthy since its binding energy in PBE calculation is $22 \mathrm{meV} /$ atom greater than that of si40-1a, while its binding energy in BLYP calculation is $21 \mathrm{meV} /$ atom greater than that of si40-1a' (Table II). Given that si40-1a and si40-1a' are the leading candidates for the lowest-energy clusters of $\mathrm{Si}_{40}$, the newly obtained si50-1a may be qualified as a good candidate for the low-lying cluster of $\mathrm{Si}_{50}$, and thus may be used as a prototype model for study chemical reaction with small chemical molecules. 
TABLE I. Optimal core-filling@ @age combinations for low-lying endohedral fullerene clusters $\mathrm{Si}_{39}$ and $\mathrm{Si}_{40}$ shown in Fig. 1. The binding energy per atom was calculated at the PWP-DFT level of theory with two GGA functionals. The boldface denotes the lowest-energy isomer (isomers whose binding energy is within $4 \mathrm{meV} /$ atom from the lowest-energy isomer are also highlighted in bold).

\begin{tabular}{|c|c|c|c|}
\hline & \multirow{2}{*}{$\begin{array}{c}\text { Optimal core-filling@cage combination } \\
\text { [homolog fullerene cage] }\end{array}$} & \multicolumn{2}{|c|}{ Binding energy (eV/atom) } \\
\hline & & PBE & BLYP \\
\hline si39-1a & $\mathrm{Si}_{5} @ \mathrm{Si}_{34}\left[\mathrm{C}_{34}\left(C_{s}: 2\right)\right]$ & 3.959 & 3.364 \\
\hline si39-1b & $\mathrm{Si}_{5} @ \mathrm{Si}_{34}\left[\mathrm{C}_{34}\left(C_{2}: 5\right)\right]$ & 3.950 & 3.357 \\
\hline si39-1c & $\mathrm{Si}_{5} @ \mathrm{Si}_{34}\left[\mathrm{C}_{34}\left(C_{3 v}: 6\right)\right]$ & 3.942 & 3.366 \\
\hline si39-1d & $\mathrm{Si}_{5} @ \mathrm{Si}_{34}\left[\mathrm{C}_{34}\left(C_{s}: 3\right)\right]$ & 3.938 & 3.358 \\
\hline si39-1e & $\mathrm{Si}_{5} @ \mathrm{Si}_{34}\left[\mathrm{C}_{34}\left(C_{2}: 4\right)\right]$ & 3.938 & 3.350 \\
\hline si39-1f & $\mathrm{Si}_{5} @ \mathrm{Si}_{34}\left[\mathrm{C}_{34}\left(C_{2}: 1\right)\right]$ & 3.929 & 3.359 \\
\hline si39 (Ref. 18) & $\mathrm{Si}_{5} @ \mathrm{Si}_{34}\left[\mathrm{C}_{34}\left(C_{s}: 2\right)\right]$ & 3.941 & 3.362 \\
\hline si39 (Ref. 17) & $\mathrm{Si}_{5} @ \mathrm{Si}_{34}\left(\mathrm{C}_{34}\right.$ has a seven-member ring $)$ & 3.939 & 3.365 \\
\hline si40-1a & $\mathrm{Si}_{6} @ \mathrm{Si}_{34}\left[\mathrm{C}_{34}\left(C_{s}: 2\right)\right]$ & 3.966 & 3.364 \\
\hline si40-1b & $\mathrm{Si}_{6} @ \mathrm{Si}_{34}\left[\mathrm{C}_{34}\left(C_{2}: 5\right)\right]$ & 3.962 & 3.365 \\
\hline si40-1c & $\mathrm{Si}_{6} @ \mathrm{Si}_{34}\left[\mathrm{C}_{34}\left(C_{2}: 4\right)\right]$ & 3.949 & 3.356 \\
\hline si40-1d & $\mathrm{Si}_{6} @ \mathrm{Si}_{34}\left[\mathrm{C}_{34}\left(C_{s}: 3\right)\right]$ & 3.946 & 3.357 \\
\hline si40-1e & $\mathrm{Si}_{6} @ \mathrm{Si}_{34}\left[\mathrm{C}_{34}\left(C_{3 v}: 6\right)\right]$ & 3.944 & 3.349 \\
\hline si40-1f & $\mathrm{Si}_{6} @ \mathrm{Si}_{34}\left[\mathrm{C}_{34}\left(C_{2}: 1\right)\right]$ & 3.938 & 3.350 \\
\hline si40 (Ref. 21) & $\mathrm{Si}_{6} @ \mathrm{Si}_{34}\left(\mathrm{C}_{34}\right.$ has a seven-member ring $)$ & 3.955 & 3.353 \\
\hline si40 (Ref. 12) & $\mathrm{Si}_{6} @ \mathrm{Si}_{34}\left[\mathrm{C}_{34}\left(C_{3 v}: 6\right)\right]$ & 3.943 & 3.359 \\
\hline si40-1a' (Ref. 17) & $\mathrm{Si}_{4} @ \mathrm{Si}_{36}\left[\mathrm{C}_{36}\left(D_{3 h}: 13\right)\right]$ & 3.928 & 3.372 \\
\hline
\end{tabular}

\section{D. $\mathrm{Si}_{60}\left(\mathrm{Si}_{12} @ \mathrm{Si}_{48}\right.$ or $\left.\mathrm{Si}_{10} @ \mathrm{Si}_{50}\right)$}

As pointed out in the Introduction, $\mathrm{Si}_{60}$ is of particular interest because of its relevance to previous studies of bucky silicon $\mathrm{Si}_{60} \cdot{ }^{28-37}$ According to the empirical rule, the optimal core-filling@cage combination for endohedral fullerene $\mathrm{Si}_{60}$ is $\mathrm{Si}_{12} @ \mathrm{Si}_{48}$ or $\mathrm{Si}_{10} @ \mathrm{Si}_{50}$. Recently, Zhao et al. reported a low-lying endohedral fullerene structure of $\mathrm{Si}_{60}$ whose outer cage is homolog to $\mathrm{C}_{48}\left(C_{2}: 199\right)$. This carbon fullerene is the lowest-energy structure among the 199 fullerene isomers of $\mathrm{C}_{48}{ }^{47}$ Zhou et al. also found that endohedral fullerenes in the form of $\mathrm{Si}_{14} @ \mathrm{Si}_{46}$ combination generally give much higher energies than $\mathrm{Si}_{12} @ \mathrm{Si}_{48}$ combination. Zhou and $\mathrm{Pan}^{21}$ utilized the compressing-liquid-silicon approach and independently confirmed that the preferred structure for $\mathrm{Si}_{60}$ is endohedral fullerene. Interestingly, the reported low-energy $\mathrm{Si}_{60}$ also has a $\mathrm{Si}_{48}$ outer cage which is homolog to $\mathrm{C}_{48}\left(D_{2}: 169\right)$. The latter is the tenth lowest-energy structure among the 199 fullerene isomers of $\mathrm{C}_{48}{ }^{47}$

The $\mathrm{Si}_{60}$ structure reported by Zhao et al. has a binding energy per atom of $3.993 \mathrm{eV}$ (PBE calculation), $5 \mathrm{meV} /$ atom greater than that of si50-1a. Thus, it may be viewed as a candidate for the low-lying cluster of $\mathrm{Si}_{60}$. Because the $\mathrm{Si}_{60}$ structure of Zhao et al. was built based on the lowest-energy $\mathrm{C}_{48}$ cage, ${ }^{22}$ we just performed a direct constrained $\mathrm{BH}$ search based on this $\mathrm{Si}_{60}$ and obtained an improved structure, si60-1a [Fig. 2(b)]. Note that the binding energy per atom (PBE calculation) of si60-1a is $3.998 \mathrm{eV}, 10 \mathrm{meV} /$ atom greater than that of si50-1a (Table II). We performed another $\mathrm{BH}$ search using an initial configuration constructed based on $\mathrm{C}_{48}\left(C_{2}: 171\right)$, which is the second lowest-energy structure among the 199 fullerene isomers of $\mathrm{C}_{48}{ }^{47}$ The obtained structure is si60-1b [Fig. 2(b)], whose binding energy per atom (PBE calculation) is nearly the same as that of si60-1a (Table II).
Additionally, we performed a BH search starting from an initial configuration of endohedral fullerene $\mathrm{Si}_{60}$ whose outer cage is homolog to carbon fullerene $\mathrm{C}_{50}\left(D_{3}: 270\right)$. Note that $\mathrm{C}_{50}\left(D_{3}: 270\right)$ is the lowest-energy structure among the 271 fullerene isomers of $\mathrm{C}_{50}{ }^{47}$ The resulting low-energy structure is named si60-1 $\mathrm{a}^{\prime}$ [Fig. 2(b)]. Its binding energy per atom is $3.977 \mathrm{eV}$ (PBE calculation; Table II). Moreover, si60-1 $\mathrm{a}^{\prime}$ has the greatest binding energy per atom, $3.421 \mathrm{eV}$, in BLYP calculation, compared to other isomers with $\mathrm{Si}_{48}$ cages. This result confirms again that the BLYP functional favors clusters with a larger fullerene cage and less corefilling atoms. In summary, si60-1a and si60-1 a' may be considered as the low-lying structures of $\mathrm{Si}_{60}$, since their binding energies per atom are at least $10 \mathrm{meV}$ greater than those of si50-1a.

\section{E. $\mathbf{S i}_{70}\left(\mathbf{S i}_{14} @ \mathrm{Si}_{56}\right.$ or $\left.\mathbf{S i}_{12} @ \mathrm{Si}_{58}\right)$}

Li et $a l .{ }^{52}$ studied an endohedral fullerene $\mathrm{Si}_{70}$ whose outer cage is homolog to the bucky ball $\mathrm{C}_{60}\left(I_{h}\right)$. They found that the endohedral fullerene cluster is much lower in energy than the fullerene cage $\mathrm{Si}_{70}$. On the other hand, according to the empirical rule, the optimal core-filling@cage combination for endohedral fullerene $\mathrm{Si}_{70}$ is $\mathrm{Si}_{14} @ \mathrm{Si}_{56}$ or $\mathrm{Si}_{12} @ \mathrm{Si}_{58}$, rather than $\mathrm{Si}_{10} @ \mathrm{Si}_{60}$.

Here, we constructed two initial configurations of endohedral fullerene $\mathrm{Si}_{70}$ using the lowest-energy carbon fullerene cages $\mathrm{C}_{56}\left(D_{2}: 916\right)$ and $\mathrm{C}_{58}\left(C_{3 v}: 1205\right)$, respectively. ${ }^{47}$ We then performed the $\mathrm{BH}$ search and obtained two low-energy structures, si70-1a and si70-1a' [Fig. 2(c)]. Both si70-1a and si70-1a' have nearly the same binding energy per atom in PBE calculation, as well as nearly degenerate binding energy per atom in BLYP calculation (Table II). Note that the binding energy per atom of si70-1a 


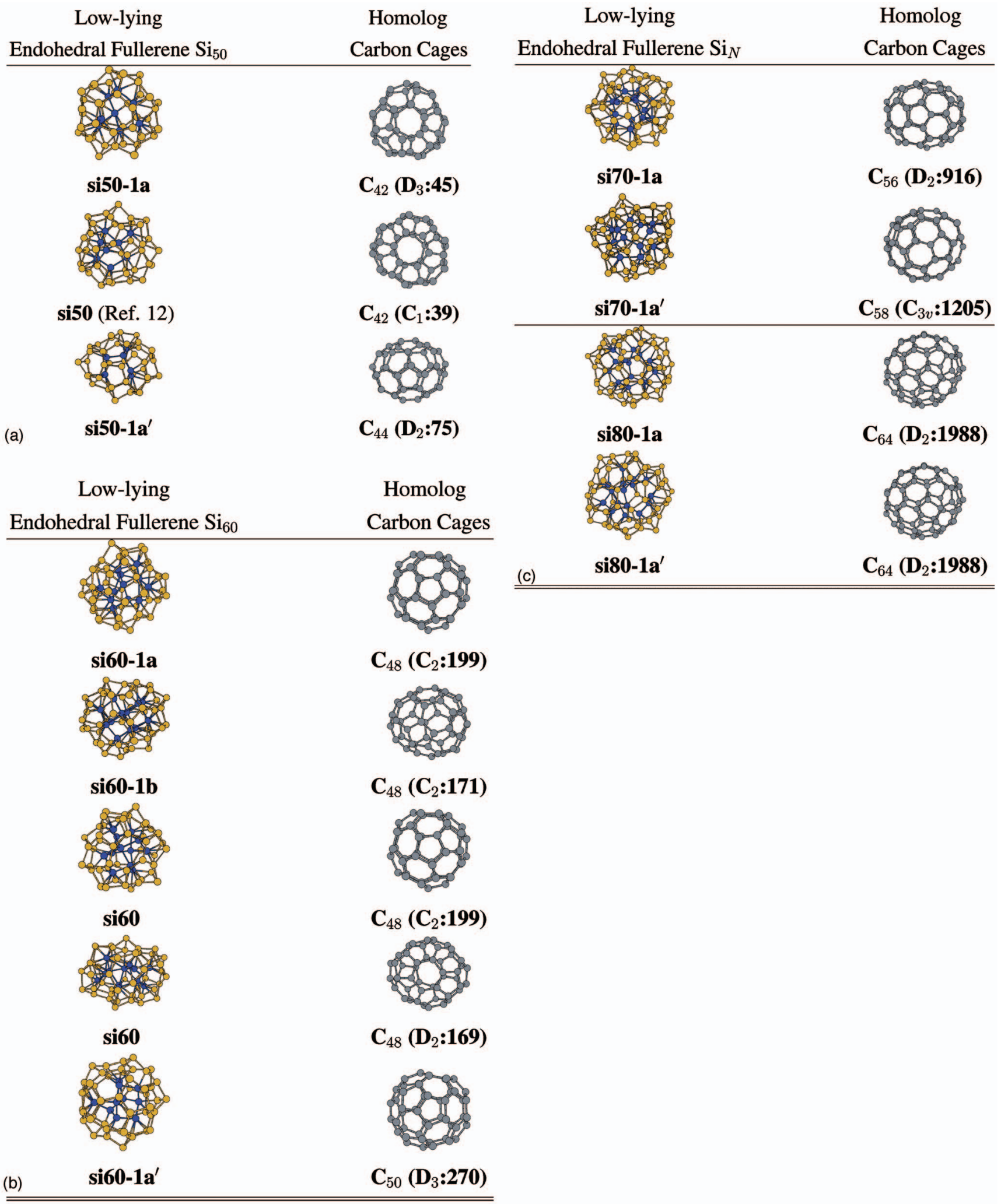

FIG. 2. (Color) Geometries of low-lying endohedral silicon fullerene clusters (a) $\mathrm{Si}_{50}$, (b) $\mathrm{Si}_{60}$, and (c) $\mathrm{Si}_{70}$ and $\mathrm{Si}_{80}$. The core-filling atoms are in blue and the outer cage atoms are in yellow. The corresponding homolog carbon fullerene cages (gray) are also displayed.

is $4.011 \mathrm{eV}$ (PBE calculation), about $13 \mathrm{meV} /$ atom greater than that of si60-1a, whereas the binding energy per atom of si70-1 $\mathrm{a}^{\prime}$ is $3.430 \mathrm{eV}$ (BLYP calculation), about $9 \mathrm{meV} / \mathrm{atom}$ greater than that of si60-1a'. Hence, si70-1a meets the minimal requirement (at least $10 \mathrm{meV} / \mathrm{atom}$ greater) to be considered as low-lying structures of $\mathrm{Si}_{70}$.
There is still room to improve the endohedral fullerene structures of $\mathrm{Si}_{70}$ to achieve even lower energy than that of si70-1a or si70-1 $a^{\prime}$. For example, one could in principle perform $\mathrm{BH}$ searches based on initial configurations constructed from all the 924 and 1205 isomers of $\mathrm{C}_{56}$ and $\mathrm{C}_{58}$ cage motifs, ${ }^{47}$ respectively, as well as those based on $\mathrm{C}_{54}$ and $\mathrm{C}_{60}$ 
TABLE II. Optimal core-filling@ cage combinations for low-lying endohedral fullereen clusters $\mathrm{Si}_{50}, \mathrm{Si}_{60}, \mathrm{Si}_{70}$, and $\mathrm{Si}_{80}$ shown in Fig. 2. The binding energy per atom was calculated at the PWP-DFT level of theory with two GGA functionals. The boldface denotes the lowest-energy isomer (isomers whose binding energy is within $4 \mathrm{meV} /$ atom from the lowest-energy isomer are also highlighted in bold).

\begin{tabular}{|c|c|c|c|}
\hline & \multirow{2}{*}{$\begin{array}{l}\text { Optimal stuffing cage combination } \\
\text { [homologfullerene cage }]\end{array}$} & \multicolumn{2}{|c|}{ Binding energy (eV/atom) } \\
\hline & & PBE & BLYP \\
\hline si50-1a & $\mathrm{Si}_{8} @ \mathrm{Si}_{42}\left[\mathrm{C}_{42}\left(D_{3}: 45\right)\right]$ & 3.988 & 3.393 \\
\hline si50 (Ref. 12) & $\mathrm{Si}_{8} @ \mathrm{Si}_{42}\left[\mathrm{C}_{42}\left(C_{1}: 39\right)\right]$ & 3.954 & 3.364 \\
\hline si50 (Ref. 21) & $\mathrm{Si}_{10} @ \mathrm{Si}_{40}\left(\mathrm{C}_{40}\right.$ has a seven-member ring $)$ & 3.950 & 3.361 \\
\hline si50-1 $a^{\prime}$ & $\mathrm{Si}_{6} @ \mathrm{Si}_{44}\left[\mathrm{C}_{42}\left(D_{2}: 75\right)\right]$ & 3.938 & 3.400 \\
\hline si60-1a & $\mathrm{Si}_{12} @ \mathrm{Si}_{48}\left[\mathrm{C}_{48}\left(C_{2}: 199\right)\right]$ & 3.998 & 3.395 \\
\hline si60-1b & $\mathrm{Si}_{12} @ \mathrm{Si}_{48}\left[\mathrm{C}_{48}\left(C_{2}: 171\right)\right]$ & 3.996 & 3.386 \\
\hline si60 (Ref. 22) & $\mathrm{Si}_{12} @ \mathrm{Si}_{48}\left[\mathrm{C}_{48}\left(C_{2}: 199\right)\right]$ & 3.993 & 3.388 \\
\hline si60 (Ref. 21) & $\mathrm{Si}_{12} @ \mathrm{Si}_{48}\left[\mathrm{C}_{48}\left(D_{2}: 169\right)\right]$ & 3.987 & 3.379 \\
\hline si60-1 $a^{\prime}$ & $\mathrm{Si}_{10} @ \mathrm{Si}_{50}\left[\mathrm{C}_{50}\left(D_{3}: 270\right)\right]$ & 3.977 & 3.421 \\
\hline si70-1a & $\mathrm{Si}_{14} @ \mathrm{Si}_{56}\left[\mathrm{C}_{56}\left(D_{2}: 916\right)\right]$ & 4.011 & 3.427 \\
\hline si70-1 $\mathrm{a}^{\prime}$ & $\mathrm{Si}_{12} @ \mathrm{Si}_{58}\left[\mathrm{C}_{58}\left(C_{3 v}: 1205\right)\right]$ & 4.007 & 3.430 \\
\hline si80-1a & $\mathrm{Si}_{16} @ \mathrm{Si}_{64}\left[\mathrm{C}_{64}\left(D_{2}: 1998\right)\right]$ & 4.025 & 3.451 \\
\hline si80-1 $a^{\prime}$ & $\mathrm{Si}_{16} @ \mathrm{Si}_{64}\left[\mathrm{C}_{64}\left(D_{2}: 1988\right)\right]$ & 4.024 & 3.452 \\
\hline
\end{tabular}

cage motifs. However, the main goal of this study is not to locate a single global minimum for each medium- or largesized silicon clusters but to obtain prototype low-lying clusters that can represent one class of low-lying endohedral silicon clusters in the considered size range (see Sec. IV and Fig. 3 below).

\section{F. $\mathrm{Si}_{80}\left(\mathrm{Si}_{18} @ \mathrm{Si}_{62}\right.$ or $\left.\mathrm{Si}_{16} @ \mathrm{Si}_{64}\right)$}

For large-sized cluster $\mathrm{Si}_{80}$, the empirical rule suggests that the optimal core-filling@cage combination is $\mathrm{Si}_{18} @ \mathrm{Si}_{62}$ or $\mathrm{Si}_{16} @ \mathrm{Si}_{64}$. We thus constructed two initial configurations of endohedral fullerene $\mathrm{Si}_{80}$ using the lowest-energy carbon fullerene cage of $\mathrm{C}_{62}\left(C_{2}: 1994\right)$ and $\mathrm{C}_{64}\left(D_{2}: 1988\right)$, respectively. ${ }^{47}$ We then performed the $\mathrm{BH}$ search and obtained two structures, si80-1a and si80-1a' [Fig. 2(c)]. Both si80-1a and si80-1a' have nearly the same binding energy per atom in PBE and BLYP calculation and both low-lying isomers are obtained based on the $\mathrm{C}_{64}\left(D_{2}: 1988\right)$ cage motif. Interestingly, the low-lying isomer obtained based on $\mathrm{C}_{62}\left(D_{2}: 1994\right)$ cage motif is slighly higher in energy than si80-1a (PBE calculation), even though si80-1a has less core filling $\mathrm{Si}$ atoms (16). It is worthy to mention that the 16 core-filling $\mathrm{Si}$ atoms in si80-1a [blue atoms in Fig. 2(c)] also forms a cage-like structure. Thus, si80-1a may be viewed as a cage-in-cage or doubleshell structure.

Note that the binding energy per atom of si80-1a is $4.025 \mathrm{eV}$ (PBE calculation), about $14 \mathrm{meV} / \mathrm{atom}$ greater than that of si70-1a, and the binding energy per atom of si80-1 $\mathrm{a}^{\prime}$ is $3.452 \mathrm{eV}$ (BLYP calculation), about $22 \mathrm{meV} /$ atom greater than that of si70-1 $\mathrm{a}^{\prime}$. Therefore, both si80-1a and si80-1 $\mathrm{a}^{\prime}$ structures meet the minimal requirement to be considered as low-lying structures of $\mathrm{Si}_{80}$.

\section{COMPARISON WITH LOWEST-ENERGY STILLINGER-WEBER SILICON CLUSTERS}

In a previous study, ${ }^{45}$ we performed an unbiased global search for the global minima of silicon clusters up to $\mathrm{Si}_{30}$ by using three empirical potential models of silicon. In general, for small-sized silicon clusters (e.g., $\mathrm{Si}_{6}-\mathrm{Si}_{20}$ ), the global minima of empirical model silicon clusters differ markedly from the true global minima of silicon clusters. However, we found that the SW potential, ${ }^{44}$ which is fitted to reproduce bulk properties of silicon, predicts the onset of endohedral spherical-like lowest-energy structures at $\mathrm{Si}_{20}$. Although, in reality, the onset of endohedral spherical-like lowest-energy structures occurs in the size range of $\mathrm{Si}_{27}-\mathrm{Si}_{30}$, it is expected that the SW potential will predict better overall structures of silicon clusters as the size of the cluster increases. Therefore, for the purpose of comparison, we also carried out an unbiased $\mathrm{BH}$ search for the global minima of $\mathrm{SW} \mathrm{Si}_{39}, \mathrm{Si}_{40}, \mathrm{Si}_{50}$, $\mathrm{Si}_{60}, \mathrm{Si}_{70}$, and $\mathrm{Si}_{80}$. Details of the method have been published in Ref. 45. For $\mathrm{SW} \mathrm{Si}_{39}, \mathrm{Si}_{40}$, and $\mathrm{Si}_{50}$, we were able to locate the global minima starting from three independent random structures, typically within $50000 \mathrm{MC}$ steps. However, for $\mathrm{SW} \mathrm{Si}_{60}, \mathrm{Si}_{70}$, and $\mathrm{Si}_{80}$, we only obtained a number of low-lying clusters after $100000 \mathrm{MC}$ steps. Results of the obtained lowest-lying or low-lying clusters of SW clusters are shown in Table III and Fig. 3. As expected, the lowestlying or low-lying SW silicon clusters from medium to large

TABLE III. Lowest-lying or low-lying isomers obtained from BH global search on the basis of the $\mathrm{SW}$ potential model. $\mathrm{SW} \mathrm{Si}_{39}, \mathrm{Si}_{40}$, and $\mathrm{Si}_{50}$ are the predicted global minima, whereas $\mathrm{SW} \mathrm{Si}_{60}, \mathrm{Si}_{70}$, and $\mathrm{Si}_{80}$ are the best local minima obtained from this study. The (classical) potential energy per atom $V / n$ is given in the dimensionless unit. We also reoptimized the isomers at the DFT-PBE and DFT-BLYP levels of theory and calculated the corresponding binding energy per atom $(\mathrm{eV})$. The bold highlights the binding energies per atom of low-lying cluster of $\mathrm{Si}_{70}$.

\begin{tabular}{ccccc}
\hline \hline $\mathrm{SW}$ & Point group & $V / n$ & $\mathrm{DFT} / \mathrm{PBE}$ & DFT/BLYP \\
\hline $\mathrm{Si}_{39}\left(\mathrm{Si}_{5} @ \mathrm{Si}_{34}\right)$ & $C_{1}$ & -1.6079 & 3.919 & 3.352 \\
$\mathrm{Si}_{40}\left(\mathrm{Si}_{4} @ \mathrm{Si}_{36}\right)$ & $C_{2 \mathrm{v}}$ & -1.6104 & 3.921 & 3.363 \\
$\mathrm{Si}_{50}\left(\mathrm{Si}_{8} @ \mathrm{Si}_{42}\right)$ & $C_{3}$ & -1.6408 & 3.961 & 3.389 \\
$\mathrm{Si}_{60}\left(\mathrm{Si}_{12} @ \mathrm{Si}_{48}\right)$ & $C_{1}$ & -1.6584 & 3.979 & 3.382 \\
$\mathrm{Si}_{70}\left(\mathrm{Si}_{14} @ \mathrm{Si}_{56}\right)$ & $C_{1}$ & -1.6715 & $\mathbf{4 . 0 1 1}$ & $\mathbf{3 . 4 3 3}$ \\
$\mathrm{Si}_{80}\left(\mathrm{Si}_{16} @ \mathrm{Si}_{64}\right)$ & $C_{1}$ & -1.6860 & 4.002 & 3.428 \\
\hline \hline
\end{tabular}




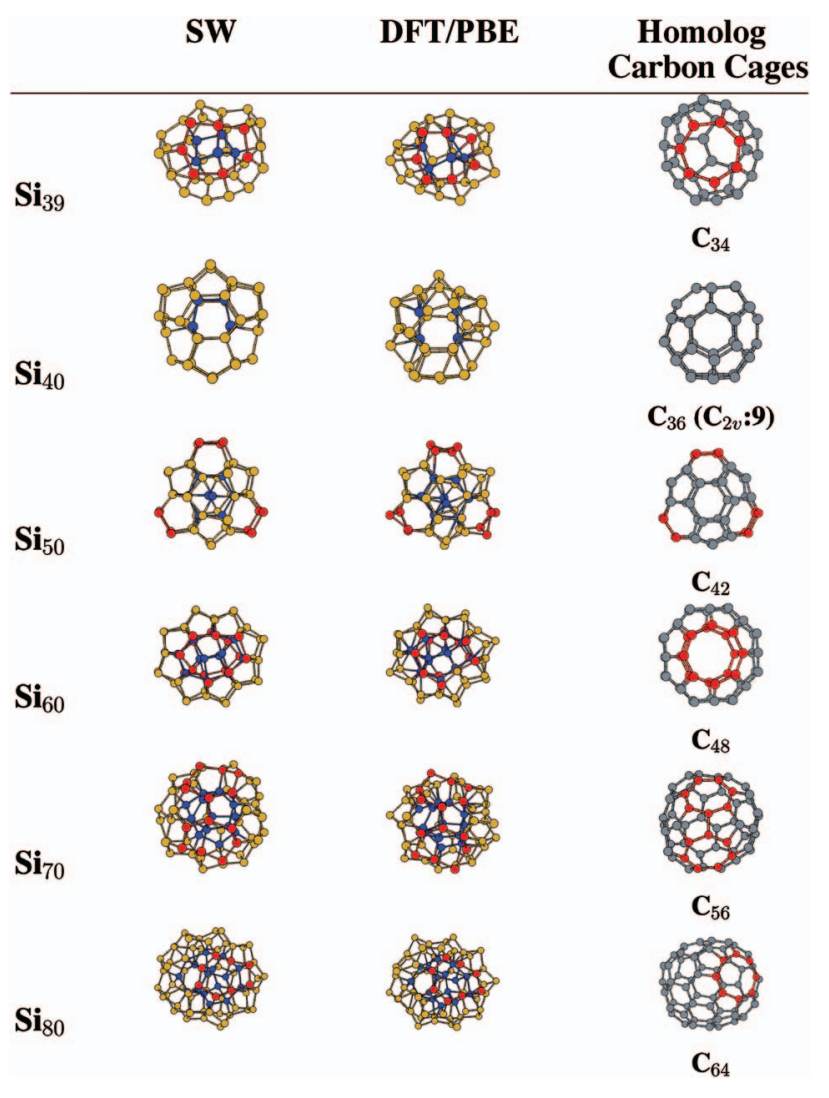

FIG. 3. (Color) Geometries of lowest-lying SW silicon clusters $\mathrm{SW} \mathrm{Si}_{39}$, $\mathrm{Si}_{40}$, and $\mathrm{Si}_{50}$, as well as the low-lying $\mathrm{SW} \mathrm{Si}_{60}, \mathrm{Si}_{70}$, and $\mathrm{Si}_{80}$. The corefilling atoms are in blue and the outer cage atoms are in yellow. The corresponding homolog carbon fullerene cages (gray) are also displayed where the four, seven, and eight members are highlighted in red.

size all exhibit spherical-like endohedral structures. Except $\mathrm{SW} \mathrm{Si} i_{40}$, the corresponding homolog carbon cages are generally nonfullerene cages. In Fig. 3, we highlight four-, seven-, and eight-membered rings on the outer cage of SW clusters.

To compare with the low-lying clusters obtained from the motif-based constrained search, we reoptimized the SW silicon clusters using the DFT-PBE and DFT-BLYP levels of theory. Results of the binding energy per atoms are also shown in Table III. It can be seen that, with the exception of $\mathrm{SW} \mathrm{Si} i_{70}$, the binding energy per atom in PBE calculations is notably less (ranging from 14 to $45 \mathrm{meV} / \mathrm{atom}$ ) than the obtained low-lying clusters shown in Tables I and II. Hence, most of these lowest-lying or low-lying SW clusters cannot be qualified as the true low-lying silicon clusters. On the other hand, $\mathrm{SW} \mathrm{Si}_{70}$ possesses binding energies per atom of $4.011 \mathrm{eV} /$ atom (PBE calculation) and $3.433 \mathrm{eV} /$ atom (BLYP calculation), which are comparable or slightly greater than those of si70-1a and si70-1 $\mathrm{a}^{\prime}$, respectively. Hence, $\mathrm{SW} \mathrm{Si}$ can be viewed as a low-lying cluster. Note that the homolog carbon cage of $\mathrm{SW} \mathrm{Si}_{70}$ is also $\mathrm{C}_{56}$ but containing two sevenmembered rings (Fig. 3). It is still remarkable to see that the unbiased search based on the SW potential also gives rise to the same optimal core-filling/cage combination $\left(\mathrm{Si}_{14} @ \mathrm{Si}_{56}\right)$ as the empirical rule predicts. This result suggests that the SW potential becomes increasingly reasonable in predicting large-sized low-energy silicon clusters.

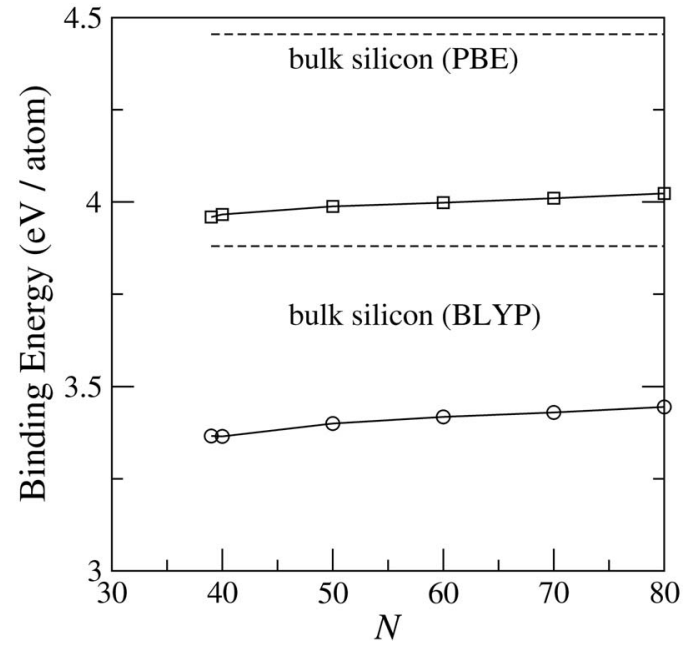

FIG. 4. Binding energies per atom (eV/atom) of the lowest-energy endohedral silicon fullerenes highlighted (in bold) in Tables I and II. The squares represent PBE binding energies while the circles represent BLYP binding energies. The two horizontal dashed lines represent cohesive energies of the bulk silicon from PBE and BLYP calculations, respectively.

\section{FURTHER DISCUSSIONS}

In Fig. 4, we plot the binding energy per atom for the obtained low-lying clusters (highlighted in bold in Tables I and II). It can be seen that for clusters larger than $\mathrm{Si}_{50}$, the binding energy per atom increases almost linearly with the size of the cluster, and the slope of the solid lines is $\sim 1-1.4 \mathrm{meV} /$ atom. In other words, with addition of one silicon atom to the cluster, the binding energy per atom (or cohesive energy) should gain at least $1 \mathrm{meV}$. We have considered this result as an empirical criterion (or the minimal requirement) to identify low-lying silicon clusters with size larger than $\mathrm{Si}_{50}$.

Jarrold and Honea have reported cohesive energies (or binding energies per atom) of cluster ions in the range of $\mathrm{Si}_{6}-\mathrm{Si}_{70}{ }^{53}$ From their data (Fig. 6 in Ref. 53), it appears that the measured cohesive energies increase linearly with the size of the clusters in the range of $\mathrm{Si}_{50}-\mathrm{Si}_{70}$. We estimated the slope of the dotted line (Fig. 6 in Ref. 53) for the largesized clusters (e.g., $\quad \mathrm{Si}_{63}-\mathrm{Si}_{70}$ ), which is about

TABLE IV. Calculated HOMO-LUMO gaps for low-lying endohedral fullerene clusters $\mathrm{Si}_{40}, \mathrm{Si}_{50}, \mathrm{Si}_{60}, \mathrm{Si}_{70}$, and $\mathrm{Si}_{80}$. The geometric optimization is performed at the PWP-DFT level of theory with the PBE functional. The HOMO-LUMO gaps are calculated based on the B3LYP/6-31G $(d)$ singlepoint calculation.

\begin{tabular}{cc}
\hline \hline & HOMO-LUMO gap $(\mathrm{eV})$ \\
\hline Si40-1a & 1.25 \\
Si40-1a' & 0.90 \\
Si50-1a & 1.42 \\
si50-1a' & 1.36 \\
Si60-1a & 1.12 \\
Si60-1a' & 1.36 \\
Si70-1a & 0.84 \\
Si70-1a' & 1.25 \\
Si80-1a & 0.74 \\
Si80-1a' & 1.01 \\
\hline
\end{tabular}


$1.3 \mathrm{meV} /$ atom. Hence, our theoretical result on the increase of cohesive energy with increasing the cluster size appears to be consistent with the measured result in the same size range, lending strong support on the empirical criterion (or minimal requirement) proposed here.

We also calculated the cohesive energy of the bulk silicon using the CPMD program, that is, $4.455 \mathrm{eV}$ in PBE calculation and $3.880 \mathrm{eV}$ in BLYP calculation (dashed lines in Fig. 4). If we assumed that the binding energy per atom increases linearly with increasing the cluster size, the two solid lines would intercept with the two dashed lines at about $N \sim 400$, suggesting that the core of the endohedral silicon cluster could be crystallinelike at the size of the cluster approaching $N=400.8,54,55$

As stated previously, the main goal of this study is to obtain a class of representative low-lying clusters that can be used for the study of chemical reaction with small chemical molecules, the growth pattern of the spherical-like silicon clusters, or the size-dependent electronic properties. ${ }^{50,54-57}$ For the latter, as an example, we have calculated highest occupied molecular orbital (HOMO)-lowest unoccupied molecular orbital (LUMO) gaps of the representative low-lying clusters (see Table IV). We used a hybrid density functional (B3LYP) with the 6-31G $(d)$ basis set. ${ }^{58}$ We find that for medium- to large-sized clusters, the HOMO-LUMO gaps are typically around $1 \mathrm{eV}$, and the gaps show a maximum at size $N=50$. Another interesting observation is that for $N \leqslant 50$, the compact clusters with more core-filling atoms (e.g., si40-1a and si50-1a) exhibit a larger HOMO-LUMO gap than the less compact isomers with less core-filling atoms (e.g., si40-1 $\mathrm{a}^{\prime}$ and si50-1 $\left.\mathrm{a}^{\prime}\right)$, whereas for $N \geqslant 60$, the less compact clusters (e.g., si60-1 $\mathrm{a}^{\prime}$, si70-1 $\mathrm{a}^{\prime}$, and si80-1 $\mathrm{a}^{\prime}$ ) possess larger energy gaps than the compact clusters (e.g., si60-1a, si70-1a, and si80-1a).

\section{CONCLUSION}

In conclusion, we have performed a constrained basinhopping search for the low-lying endohedral silicon clusters in the medium-to-large size range. For $\mathrm{Si}_{39}, \mathrm{Si}_{40}$, and $\mathrm{Si}_{50}$, the $\mathrm{BH}$ search indicates that clusters constructed based on the fullerene cage motifs consistently achieve lower energy than those based on nonfullerene cage motifs (previously obtained from unconstrained global search). Thus, this study further confirms that fullerene cages are promising cage motifs for building low-lying spherical-like clusters, at least up to $\mathrm{Si}_{50}$ and possibly up to $\mathrm{Si}_{60}$. For $\mathrm{Si}_{70}$, we found two candidates of low-lying cluster, one with a fullerene cage and the best one with a nonfullerene cage containing two sevenmembered rings. The latter isomer was obtained based on an unbiased search using the empirical Stillinger-Weber potential of silicon. Interestingly, the unbiased search based on the SW potential also gives rise to the same optimal corefilling@cage combination $\left(\mathrm{Si}_{14} @ \mathrm{Si}_{56}\right)$ as an empirical rule suggests. This result suggests that the SW potential becomes increasingly reasonable in predicting large-sized low-energy silicon clusters. Finally, we remark that the obtained lowlying silicon clusters $\mathrm{Si}_{50}$ and $\mathrm{Si}_{60}$ and possibly $\mathrm{Si}_{70}$ and $\mathrm{Si}_{80}$ can be useful as prototype structural models to study sizedependent properties.

\section{ACKNOWLEDGMENTS}

We are grateful to valuable discussions with Professor Th. Frauenheim, Professor B.C. Pan, Professor J. Zhao, and Professor R.L. Zhou. This work is supported by grants from the DOE (DE-FG02-04ER46164), NSF (CHE-0427746), the Nebraska Research Initiative, and by the Research Computing Facility at University of Nebraska-Lincoln.

${ }^{1}$ D. A. Jelski, B. L. Swift, T. T. Rantala, X. Xia, and T. F. George, J. Chem. Phys. 95, 8552 (1991).

${ }^{2}$ E. Kaxiras and K. Jackson, Phys. Rev. Lett. 71, 727 (1993).

${ }^{3}$ U. Rothlisberger, W. Andreoni, and M. Parrinello, Phys. Rev. Lett. 72, 665 (1994).

${ }^{4}$ J. Pan and M. V. Ramakrishna, J. Chem. Phys. 101, 8108 (1994).

${ }^{5}$ M. Menon and K. R. Subbaswamy, Phys. Rev. B 51, 17952 (1995).

${ }^{6}$ E. Kaxiras, Phys. Rev. Lett. 64, 551 (1990); Phys. Rev. B 56, 13455 (1997).

${ }^{7}$ Q. Sun, Q. Wang, P. Jena, S. Waterman, and Y. Kawazoe, Phys. Rev. A 67, 063201 (2003).

${ }^{8}$ A. Sieck, Th. Frauenheim, and K. A. Jackson, Phys. Status Solidi B 240, 537 (2003).

${ }^{9}$ S. Yoo, J. J. Zhao, J. L. Wang, and X. C. Zeng, J. Am. Chem. Soc. 126, 13845 (2004).

${ }^{10}$ N. N. Nair, T. Bredow, and K. Jug, J. Comput. Chem. 25, 1255 (2004).

${ }^{11}$ S. Ma, G. Wang, J. Wang, and L. Xue, Phys. Lett. A 337, 216 (2005).

${ }^{12}$ J. Zhao, J. Wang, J. Jellinek, S. Yoo, and X. C. Zeng, Eur. Phys. J. D 34, 35 (2005)

${ }^{13}$ S. Yoo and X. C. Zeng, J. Chem. Phys. 123, 164303 (2005).

${ }^{14}$ J. Wang, X. Zhou, G. Wang, and J. Zhao, Phys. Rev. B 71, 113412 (2005).

${ }^{15}$ B.-X. Li, Phys. Rev. B 71, 235311 (2005).

${ }^{16}$ J. Bai, L.-F. Cui, J. Wang, S. Yoo, X. Li, J. Jellinek, C. Koehler, T. Frauenheim, L.-S. Wang, and X. C. Zeng, J. Phys. Chem. A 110, 908 (2006).

${ }^{17}$ S. Yoo and X. C. Zeng, J. Chem. Phys. 124, 054304 (2006); S. Yoo, N. Shao, C. Koehler, Th. Frauenheim, and X. C. Zeng, ibid. 124, 164311 (2006).

${ }^{18}$ L. Ma, J. Zhao, J. Wang, B. Wang, and G. Wang, Phys. Rev. A 73, 063203 (2006).

${ }^{19}$ R. L. Zhou and B. C. Pan, Phys. Rev. B 73, 045417 (2006).

${ }^{20}$ O. Ona, V. E. Bazterra, M. C. Caputo, J. C. Facelli, P. Fuentealba, and M. B. Ferraro, Phys. Rev. A 73, 053203 (2006).

${ }^{21}$ R. L. Zhou and B. C. Pan, Phys. Lett. A 368, 396 (2007).

${ }^{22}$ J. Zhao, L. Ma, and B. Wen, J. Phys.: Condens. Matter 19, 226208 (2007).

${ }^{23}$ K.-M. Ho, A. A. Shvartsburg, B. Pan, Z.-Y. Lu, C.-Z. Wang, J. G. Wacker, J. L. Fye, and M. F. Jarrold, Nature (London) 392, 582 (1998).

${ }^{24}$ D. J. Wales and H. A. Scheraga, Science 285, 1368 (1999).

${ }^{25}$ I. Rata, A. A. Shvartsburg, M. Horoi, Th. Frauenheim, K. W. M. Siu, and K. A. Jackson, Phys. Rev. Lett. 85, 546 (2000).

${ }^{26}$ K. A. Jackson, M. Horoi, I. Chaudhuri, T. Frauenheim, and A. A. Shvartsburg, Phys. Rev. Lett. 93, 013401 (2004).

${ }^{27}$ S. Yoo and X. C. Zeng, Angew. Chem., Int. Ed. 44, 1491 (2005).

${ }^{28}$ S. Nagase and K. Kobayashi, Chem. Phys. Lett. 187, 291 (1991).

${ }^{29}$ F. S. Khan and J. Q. Broughton, Phys. Rev. B 43, 11754 (1991).

${ }^{30}$ M. C. Piqueras, R. Crespo, E. Orti, and F. Tomas, Chem. Phys. Lett. 213, 509 (1993).

${ }^{31}$ M. Menon and K. R. Subbaswamy, Chem. Phys. Lett. 219, 219 (1994).

${ }^{32}$ X. G. Gong and Q. Q. Zheng, Phys. Rev. B 52, 4756 (1995).

${ }^{33}$ J. Song, S. E. Ulloa, and D. A. Drabold, Phys. Rev. B 53, 8042 (1996).

${ }^{34}$ J. Leszczynski and I. Yanov, J. Phys. Chem. A 103, 396 (1999).

${ }^{35}$ B.-X. Li, P.-L. Cao, and D.-L. Que, Phys. Rev. B 61, 1685 (2000).

${ }^{36}$ Z. Chen, H. Jiao, G. Seifert, A. H. C. Horn, D. Yu, T. Clark, W. Thiel, and P. v. R. Schleyer, J. Comput. Chem. 24, 948 (2003).

${ }^{37}$ Y. Pei, Y. Gao, and X. C. Zeng, J. Chem. Phys. 127, 044704 (2007).

${ }^{38}$ S. Goedecker, J. Chem. Phys. 120, 9911 (2004).

${ }^{39}$ D. Porezag, Th. Frauenheim, Th. Köhler, G. Seifert, and R. Kaschner, Phys. Rev. B 51, 12947 (1995). 
${ }^{40}$ A. D. Becke, Phys. Rev. A 38, 3098 (1988); J. P. Perdew, Phys. Rev. B 33, 8822 (1986); C. Lee, W. Yang, and R. G. Parr, ibid. 37, 785 (1988)

${ }^{41}$ J. P. Perdew, K. Burke, and M. Ernzerhof, Phys. Rev. Lett. 77, 3865 (1996).

${ }^{42}$ J. Hutter, A. Alavi, T. Deutsch, M. Bernasconi, S. Goedecker, D. Marx, M. Tuckerman, and M. Parrinello, CPMD, Version 3.7.1, MPI für Festkörperforschung Stuttgart, 2001.

${ }^{43}$ D. J. Wales and H. A. Scheraga, Science 285, 1368 (1999); J. P. K. Doye and D. J. Wales, New J. Chem. 22, 733 (1998).

${ }^{44}$ F. H. Stillinger and T. A. Weber, Phys. Rev. B 31, 5262 (1985)

${ }^{45}$ S. Yoo and X. C. Zeng, J. Chem. Phys. 119, 1442 (2003).

${ }^{46}$ X. Zhu and X. C. Zeng, J. Chem. Phys. 118, 3558 (2003).

${ }^{47}$ X. Zhu, X. C. Zeng, Y. Lei, and B. Pan, J. Chem. Phys. 120, 8985 (2004).

${ }^{48}$ P. W. Fowler and E. D. Manolopoulos, An Atlas of Fullerenes (Clarendon, Oxford, UK, 1995).
${ }^{49}$ N. Shao, Y. Gao, and X. C. Zeng, J. Phys. Chem. C 111, 17671 (2007); N. Shao, N. Shao, Y. Gao, S. Yoo, W. An, and X. C. Zeng, J. Phys. Chem. A 110, 7672 (2006).

${ }^{50}$ J. L. Elkind, J. M. Alford, F. D. Weiss, R. T. Laaksonen, and R. E. Smalley, J. Chem. Phys. 87, 2397 (1987); S. Maruyama, L. R. Anderson, and R. E. Smalley, ibid. 93, 5349 (1990).

${ }^{51}$ M. Menon and K. R. Subbaswamy, Phys. Rev. B 50, 11577 (1994).

${ }^{52}$ B.-X. Li, J.-H. Liu, and S.-C. Zhan, Eur. Phys. J. D 32, 59 (2005).

${ }^{53}$ M. F. Jarrold and E. C. Honea, J. Phys. Chem. 95, 9181 (1991).

${ }^{54}$ T. Bachels and R. Schäfer, Chem. Phys. Lett. 324, 365 (2000).

${ }^{55}$ D. K. Yu, R. Q. Zhang, and S. T. Lee, Phys. Rev. B 65, 245417 (2002).

${ }^{56}$ H. Hofmeister, J. Dutta, and H. Hofmann, Phys. Rev. B 54, 2856 (1996).

${ }^{57}$ L. Tsetseris, G. Hadjisavvas, and S. T. Pantelides, Phys. Rev. B 76, 045330 (2007).

${ }^{58}$ M. J. Frisch, G. W. Trucks, H. B. Schlegel et al., Gaussian 03, Revision C.02, Gaussian, Inc., Wallingford, CT, 2004. 